\title{
Controlling the Usability Evaluation Process under Varying Defect Visibility
}

\author{
Martin Schmettow \\ Passau University, Information Systems II \\ 94032 Passau, Germany \\ schmettow@web.de
}

\begin{abstract}
In cases where usability is a mission critical system quality it is becoming essential to know whether an evaluation study has identified the majority of existing defects. Previous work has shown that procedures for estimating the progress of evaluation studies have to account for variation in defect visibility; otherwise, harmful bias will happen. Here, a statistical model is introduced for estimating the number of not-yet-identified defects in a study. This approach also supports exact confidence intervals and can easily be adapted to estimate the required number of sessions. The method is evaluated and shown to, in most cases, provide accurate measures. A running example illustrates how practitioners may track the progress of their studies and make quantitatively informed decisions on when to finish.
\end{abstract}

\section{Author Keywords}

Usability Evaluation, Usability Business, Process Control, Reliability, Count Data Models, Maximum Likelihood

\section{ACM Classification Keywords}

H.5.2 User Interfaces (e.g. HCI): Evaluation/methodology

\section{MOTIVATION}

Usability Evaluation in the software development process is a crucial activity to ensure the quality of the user experience. Especially formative evaluations apply for finding and fixing usability defects in the interface. With modern web and e-commerce applications this is increasingly becoming mission-critical: a single usability defects can have severe economical drawbacks, like decreased conversion rates or losing customers to competitors. When clients of usability agencies start taking this seriously this may have two effects on the usability business: First, the market demand for usability services may increase. On the downside, usability professionals may also face being taken under stronger contractual obligations. Clients may start asking for guarantees, like $99 \%$ of defects need to be discovered, and they may also expect proofs of contract fulfillment. Consequently, reliable planning, controlling and measuring Usability evaluation

(c) The Author 2009.

Published by the British Computer Society processes can become central challenges for future business models of usability agencies.

\section{INTRODUCTION}

Costs for evaluation studies are rather low compared to other development activities; but still they are under the economical trade-off of costs versus benefit. The paradigm of discount usability suggests a lax strategy, where little usability is better than no evaluation at all [10]. Without doubt, this paradigm does not apply when usability is critical for business success. In those cases reliable quantitative approaches are needed in order to balance costs and benefits. Costs for running an evaluation study could easily be estimated from experience with previous projects, presumed one knows how many users suffice to find most of the defects.

Estimating the number of required users has been tackled by several authors. Basically, there are two problems to solve. First, a proper mathematical model needs to be defined and tested. And second, the effectiveness of a particular study has to be determined in advance. A model to predict the evaluation process was first presented by Virzi [18]. It explains the progress of finding new defects depending on a general detection probability and the number of sessions ${ }^{1}$. Following a similar approach, Nielsen and Landauer found considerable differences in average detection probabilities between studies [11]. Consequently, determining the effectiveness of a particular study in advance can at best be a rough guess. Lewis found a partial solution to this problem: He proposed a procedure to calculate an estimator for the detection probability in early sessions and extrapolate the rest of the process [8].

In a recent work this mathematical model was found to be inappropriate [13]: It was shown that detection probability not only varies between study, but also between individual defects inside a study. Unfortunately, when defect visibility varies, the progress of the evaluation process decelerates in particular, the rate of newly identified defects per session is considerable lower than to be expected under the model of Virzi. An alternative mathematical model was proposed that incorporates heterogeneous visibility of defects and was shown to better explain the process outcome. However, this model was not appropriate for practical purposes - it did not

\footnotetext{
${ }^{1}$ In the following, session refers to a single participant doing an evaluation. This is usually a test person in think-aloud studies. It may also be an expert doing an inspection, e.g. in experimental comparisons of evaluation methods.
} 
allow for estimating the effectiveness in early sessions and extrapolate the process.

Previous works on process extrapolation focused on drawing the progress curve of the Virzi model in order to estimate the number of participants required for a specific goal. In this work, the focus is on controlling the process. Controlling here means deciding whether to proceed the study with further sessions or to finish it. A natural criterion for this kind of decision is the number of defects that have not been identified, yet. Such a model is introduced and evaluated in the remaining paper. It is demonstrated, that this model also applies to the problem of projecting the process.

The statistical procedure is then evaluated with a couple of data sets reported in the literature. It is shown that under certain conditions the estimator for remaining defects is accurate and reliable. The practical use of this approach is illustrated in a running example.

\section{MATHEMATICAL BACKGROUND}

This section first reviews the mathematical reasoning behind the well-known geometric series model. Then an alternative model is derived and justified that accounts for varying visibility of defects. Based on this model a procedure to estimate the number of remaining defects is introduced. Furthermore, the statistical concepts of confidence intervals and model selection are briefly explained. Finally, it is demonstrated how this model also applies for predicting the future progress of a running evaluation study.

\section{The Geometric Series model}

The first model for the progress of finding new defects in an evaluation study was proposed by Virzi [18]. This model describes the progress as a geometric series, which matches the cumulative function of the geometric distribution $\mathrm{cdf}_{\mathrm{Geom}}$, where process outcome $O$ (percent of defects discovered) depends on a basic detection probability $p$ and the number of sessions $n$ :

$$
O=\operatorname{cdf}_{\mathrm{Geom}}(n, p)=1-(1-p)^{n}
$$

The original aim was to estimate the number of participants $n$ required to discover a certain rate of defects. With the cdf $_{\text {Geom }}$ this is achieved by solving eq. 1 to $n$ :

$$
n=\frac{\log (1-O)}{\log (1-p)}
$$

Outcome $O$ is usually preset as a goal for the process (e.g. identifying $80 \%$ of all defects), but also $p$ is required to solve eq. 2. This is problematic, as Nielsen and Landauer have found the basic detection probability ranging far from .12 to .58 between studies. A compromise to this problem has been found by Lewis suggesting to estimate $p$ from the first few sessions in the study [7]. A naive approach was to estimate $p$ as the ratio of all successful detection events $D^{+}$and the number of all detection trials, which is $n \cdot d^{+}$( $n$ the number of sessions, $d^{+}$the number of defects discovered so far).

$$
p_{\text {naive }}=\frac{D^{+}}{n \cdot d^{+}}
$$

Later, Lewis found that for small sample sizes this estimator is biased towards overestimation of $p$, because the number of defects not yet discovered $d^{=0}$ are not regarded for the probability mass [8]. In other words: $d^{+}$is not the true total number of defects, it is smaller and eq. 3 will calculate $p$ too large. Lewis suggested a correction term known as the GoodTuring (GT) adjustment, which simply takes the number of unobserved defects $d^{=0}$ to be equal to those observed exactly once. When $d^{=1}$ is the number of once detected defects and $d^{+}$the number of all detected defects a GT adjusted estimator for the basic probability is:

$$
p_{G T}=\frac{p_{\text {naive }}}{1+\frac{d^{=1}}{d^{+}}}=\frac{D^{+}}{\left(1+\frac{d^{=1}}{d^{+}}\right) n d}
$$

In general, the geometric series model shares the assumptions of the widely known binomial model: The underlying stochastic process consists of a series of independent Bernoulli trials with the same probability of success. The only difference is that the geometric distribution determines the number of unsuccessful trials preceding a successful event, whereas the binomial distribution tells the expected number of successes, given the number of trials. As will be seen later, the binomial model (and its derivatives introduced in the next section) may be used to estimate the number of remaining defects. In contrast, the geometric model (and its derivative) applies when process projection, i. e. estimating the required number of sessions, is at stake. But, as will be demonstrated, it can easily be reduced to the binomial model.

\section{The Logit-Normal Binomial model}

A recent study applied the method of statistical model selection to the question whether the binomial model is appropriate for the process of defect discovery. It turned out that the basic assumption of the binomial model, all defects having the same probability to be discovered, does not hold [13]. It was shown that, in fact, defects vary widely in their visibility. A particularly crucial finding was that defect heterogeneity decelerates the process, so that the geometric series model is prone to harmful overestimation of process outcome. It was shown that an alternative statistical model, allowing $p$ to vary across defects, provides a much better prediction of the process. This was achieved by taking $p$ as a random variable following a beta distribution. The beta distribution was chosen because of it's special properties: It ranges from 0 to 1 , which is appropriate as $p$ is a probability. It has two parameters and can take a variety of shapes with different means and variances. These parameters are usually estimated from the marginal sum (number of times each defect is discovered) with the maximum likelihood method. 

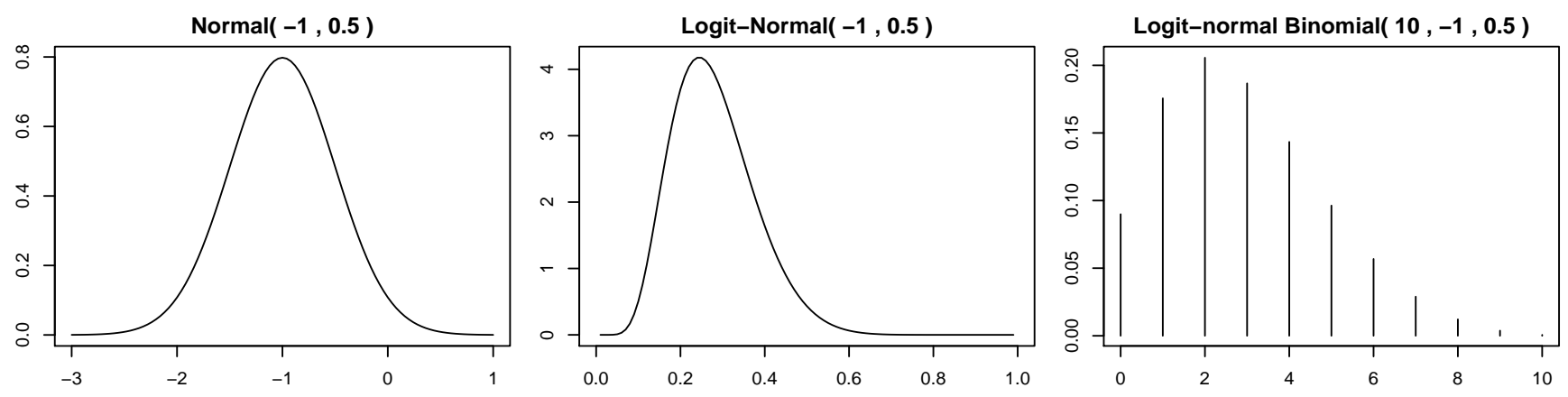

Figure 1. Derivation of logit-normal binomial distribution. The normal distribution (left) is "squeezed" into the interval $[0 ; 1]$ with the logit transformation (middle). The resulting logit-normal distribution serves as a prior for the binomial $p$ resulting in the LNB distribution (right).

Here, a similar approach is followed, but a slightly different distribution for $p$ is chosen: the logit-normal (LN) distribution. Like the beta distribution it has two parameters $\mu$ and $\sigma^{2}$ and can take a variety of shapes. In fact, both distributions are nearly indistinguishable by their shape [6]; still there were three arguments to prefer the LN distribution to the beta distribution: (1) In several pilot trials of modelling evaluation process data the LN fits similar well compared to the beta distribution, but yielded narrower confidence intervals for the parameters of interest (especially the number of remaining defects). (2) The parameters $\mu$ and $\sigma^{2}$ have a very natural interpretation: Basically, it is assumed that there is a visibility property of defects being $N\left(\mu, \sigma^{2}\right)$ normally distributed ${ }^{2}$ (see left fig. 1). The logit serves as a link function for "squeezing" the visibility property into the desired interval $[0 ; 1]$ for $p$ (fig. 1, middle). The logit transformation itself has a natural interpretation in this context in that it is the logarithm of an odd: Literally, you bet on the detection of a defect based on it's visibility. And (3) in a recent work Schmettow and Vietze introduce the Rasch model from psychometric test theory to the problem of measuring evaluation processes [16]. The logit is the inverse of the logistic function in the Rasch model - thus, both mathematical models are fully compatible.

Taking the LN distribution as a prior for the probability $p$ of the binomial distribution (fig. 1, right) results in the logitnormal binomial distribution (LNB). This distribution takes four parameters: the two unknowns $\mu$ and $\sigma^{2}$ for the distribution of $p, x$ for the observed number of successful detections and $n$ for the number of trials (independent sessions). The LNB probability distribution function is shown in eq 5 .

$$
\begin{aligned}
& \operatorname{pdf}_{L N B}\left(x ; n, \mu, \sigma^{2}\right)= \\
& \quad\left(\begin{array}{l}
n \\
x
\end{array}\right) \frac{1}{\sqrt{2 \pi \sigma^{2}}} \int_{0}^{1}(1-p)^{n-x-1} p^{x-1} \mathrm{e}^{-\frac{(\operatorname{logit}(p)-\mu)^{2}}{2 \sigma^{2}}} d p
\end{aligned}
$$

This function is mathematically complex, i.e. it does not reduce to a closed form and instead must be computed numerically (which is easy with current mathematical software

\footnotetext{
${ }^{2}$ The author has applied a Rasch analysis to a few data sets and found defect visibility to be approximately bell-shaped.
}

unless values for $\mu$ and $\sigma^{2}$ are extreme). A few relevant properties of the LNB are as follows:

- LNB mean increases with $\mu$

- LNB variance increases with $\sigma^{2}$ and is largest for $\mu=0$

- with $\sigma^{2} \rightarrow 0$ the distribution approaches the binomial distribution with basic probability $p=1 / 1+\exp (-\mu)$

\section{Defect Heterogeneity and Unseen Events}

The LNB appears appropriate to model the times defects are detected when defect visibility varies. But, there is still one problem to solve: The number of defects that have never been observed is usually unknown. For the purpose of projecting the progress of a study, Lewis applied the Good-Turing adjustment as a kind of data smoothing to estimate an unbiased binomial parameter $p$. (see eq 4). Here in contrast, the focus is on an exact estimate for the number of remaining defects $d^{=0}$. The Good-Turing adjustment provides a very good smoothing for the binomial model, but the binomial model itself is not appropriate in the presence of varying defect visibility. This fact is illustrated in fig. 2: In the left graph detection probability is invariant with $p=.28$ (i.e. it is binomially distributed, which is the limiting case for $\mu=-1$ and $\sigma^{2} \mapsto 0$ ). The two graphs to the right show what happens when variance of defect visibility increases to $\sigma^{2}=.5$ and $\sigma^{2}=1$. As expected, the distribution is getting broader and in effect $d^{=0}$ increases. Obviously, the binomial model would significantly underestimate the number of remaining defects. In the next section a modification to the LNB models is introduced which yields reliable estimators for $d^{=0}$. As another aspect it does not suffice to have a point estimator for $d^{=0}$, but also confidence intervals are required in order to make qualified decisions whether to proceed with a study. The topic of confidence intervals will be treated in a later section.

\section{The zero-truncated model}

The problem summarizes as follows: We shall fit an observed frequency distribution to a probability distribution (e.g. the binomial distribution), but one data point is missing - the number of defects that have never been observed. If one neglects this missing data, the estimation procedure "assumes" that there were no defects never observed - this results in 

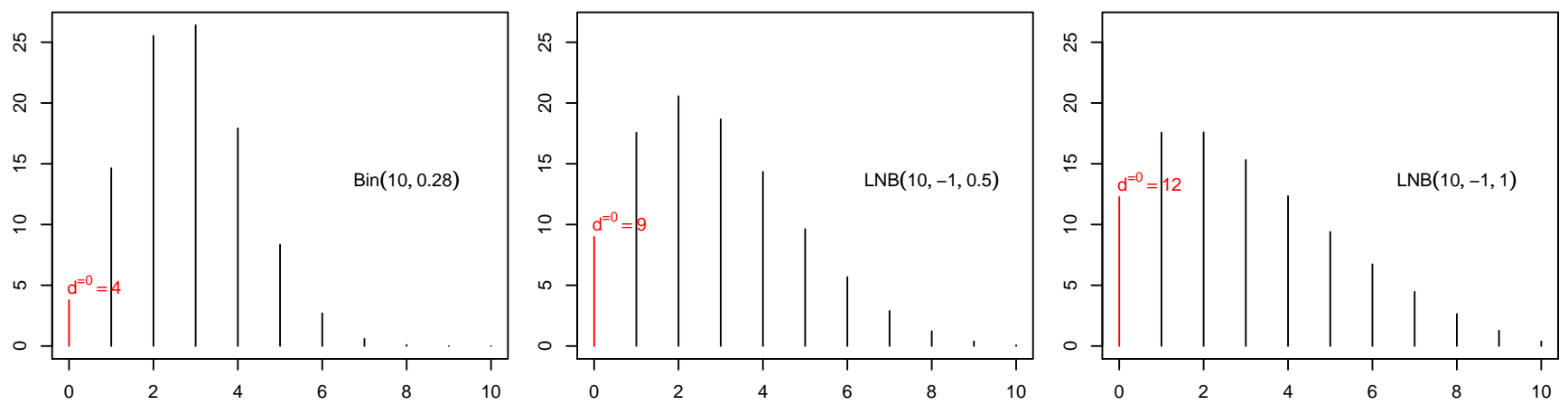

Figure 2. With increasing variance of the prior distribution the number of remaining defects $d^{=0}$ increases although the mean is held fixed.

an overly optimistic result. The Good-Turing adjustment is merely an approximation for smoothing the data at point zero. Here, a mathematically exact adjustment is introduced, which applies well for all count data distributions.

This is based on the concept of the so-called zero-truncation of probability distributions (also referred to as positive distributions). The construction of a zero-truncated probability distribution function (pdf) with arbitrary parameters $\pi$ - is rather straightforward. The number of times any defect is detected is a discrete random variable $X \in\{0, \ldots, n\}$ distributed as $P(X=x \mid \pi \cdot)=\operatorname{pdf}(x ; \pi \cdot)(\pi \cdot$ being the model parameters). Then the zero-truncated $\mathrm{pdf}_{\mathrm{zt}}$ is achieved by setting the probability of counts with $X=0$ to zero and readjusting the probability mass to 1 :

$$
\operatorname{pdf}_{z t}(x ; \pi \cdot)= \begin{cases}0 & x=0 \\ \frac{\operatorname{pdf}(x ; \pi \cdot)}{1-\operatorname{pdf}(0 ; \pi \cdot)} & x>0\end{cases}
$$

The parameters $\pi$. of a zero-truncated $\operatorname{pdf}_{z t}(x, \pi \cdot)$ are then estimated via maximum likelihood method in the usual way by defining the likelihood function $L\left(X_{1}, \ldots, X_{n} \mid \pi \cdot\right)$ and choosing $\pi$. at the point $L \max$, where $L$ has its global maximum. Finally, the expected rate of still unobserved defects can easily be estimated with the original (non-truncated) pdf and the estimated parameters $\hat{\pi} \cdot$ :

$$
P(X=0 \mid \pi \cdot)=\operatorname{pdf}(0 ; \hat{\pi} \cdot)
$$

Zero-truncation applies to any count data model, irrespectively it being the binomial distribution, the beta-binomial or the LNB. The zero-truncated Binomial distribution $\left(\operatorname{Bin}_{z t}\right)$ relates to the geometric series model and is possibly a replacement for the small sample adjustments by Lewis. But, remember that the $\mathrm{Bin}_{z t}$ model is likely to estimate a too fast progress and the $\mathrm{LNB}_{z t}$ is suggested as an alternative here.

The procedure for estimating the number of remaining defects with $\mathrm{LNB}_{z t}$ is now as simple as the following four steps: Consider a usability testing study consisting of $n=10$ sessions and $d_{n}=100$ defects have been discovered so far.
1. Prepare the data vector $D$ by calculating the number of detection events per defect

$$
\Longrightarrow \begin{array}{l|cccccc}
\text { Defect } & 1 & 2 & 3 & \ldots & 99 & 100 \\
\hline \text { Detected } & 5 & 4 & 6 & \ldots & 1 & 1
\end{array}
$$

2. Estimate the parameters $\mu$ and $\sigma^{2}$ of the zero truncated $\mathrm{LNB}_{z t}$ distribution using maximum likelihood estimation $\Longrightarrow \hat{\mu}=-1.2$ and $\hat{\sigma}^{2}=0.7$

3. Estimate the proportion of remaining defects $p^{=0}$ by using the estimated parameters with the non truncated LNB distribution

$\Longrightarrow \hat{p}^{=0}=\operatorname{LNB}\left(0 ; \hat{\mu}=-1.2, \hat{\sigma}^{2}=0.7, n=10\right)=0.12$

4. Scale with the number of identified defects $d_{n}$ $\Longrightarrow \hat{d}^{=0}=\frac{d_{n}}{1-\hat{p}^{=0}}-d_{n}=\frac{100}{0.88}-100 \approx 14$ defects remain undiscovered

\section{Computing confidence intervals}

Assume a study where the number of remaining defects is continuously tracked in order to satisfy a certain goal of $90 \%$ defects being discovered. Imagine that after 12 sessions 90 defects have been discovered and the estimation is $\hat{d}^{=0}=9$. It appears that slightly more than $90 \%$ have been discovered and the study may finish. This neglects that estimators for random processes are always random themselves. In the above case the estimation can only be interpreted as " 9 is the most likely number of remaining defects", but the true value may be a little smaller or larger. What you actually want is to make statements like "with a confidence of $95 \%$ a proportion of at least $90 \%$ of the defects has been discovered", where "confidence of $95 \%$ " has the meaning that the probability to err is only $5 \%$.

Confidence intervals are used to determine a range in which the true value lies with a certain probability, but computing them for an estimator is not trivial. Only in very special cases, like the mean of a normally distributed variable, confidence intervals can be computed straight forward. In general, there are at least three alternatives for calculation: Assuming them to be normally distributed (which is only asymptotically the case for maximum likelihood estimators), using the Fisher information function (which is often difficult to obtain) or bootstrapping. The method of bootstrapping is used here. It is rather easy to comprehend and does not make critical assumptions like normality. A nice introduction to the topic 
can be found in [9]. As a drawback, bootstrapping is computing intensive, but this is negligible with modern computers. The procedure is straight forward: One takes many repeated samples with (sic!) replacement from the original data and each time compute the maximum likelihood estimators. The result is a distribution of estimators where one can determine the standard error or confidence intervals.

Please note, that for deciding whether a study is to continue, the upper confidence limit is of particular importance. An upper confidence limit of $95 \%$ tells that the true value is greater than the limit with a probability of only $5 \%$. This is comparable to a one-sided statistical test. However, in the later evaluation both confidence limits $5 \%$ and $95 \%$ will usually be reported to give an idea of how reliable the estimator for remaining defects is. (Also note, that this results in a $90 \%$ confidence interval.)

\section{Model Selection}

It was already shown for several data sets that the heterogeneous beta-binomial model fits the defect margin sum (the number of sessions each defect was identified in) better [13]. The same approach of statistical model selection applies to whether the fit of the zero-truncated LNB model is better than the zero-truncated binomial model. Again, maximum likelihood estimation together with the Akaike Information Criterion (AIC) is used to compare models that differ in their number of parameters. The AIC penalizes the number of parameters in a model and therefore adheres to the principle of parsimony expressed by the well known Occam's Razor [2].

\section{A note on projecting the process}

Although this work focuses on estimating the number of remaining defects and not on process projection, there is a tight connection between both. In fact, the problem of drawing the curve of progress can be reduced to estimating $P(X=0)$ of the marginal sum distribution. This is easily demonstrated for the binomial distribution:

$$
\operatorname{pdf}_{\operatorname{Binom}}(x ; n, p)=\left(\begin{array}{l}
n \\
x
\end{array}\right) p^{x}(1-p)^{n-x}
$$

Setting $x=0$ reduces this to $(1-p)^{n}$ and by comparing this to the function of the geometric series in eq. 1 we get the following relation:

$$
\operatorname{cdf}_{\operatorname{Geom}}(n, p)=1-\operatorname{pdf}_{\operatorname{Binom}}(0 ; n, p)
$$

Apparently, projecting the process size is as simple as modifying step 3 above by setting $n$ to a deliberate value. For example, if the proportion of discovered defects after 20 sessions is of interest, compute $\operatorname{LNB}(0 ;-1.2,0.7, \mathbf{n}=\mathbf{2 0})=0.03$. This predicts that with 20 sessions as much as $97 \%$ of defects will have been discovered.

\section{EVALUATION}

In the following the zero-truncated LNB model is evaluated with four data sets that have been reported in the HCI literature. The evaluation is conducted to clarify the following research questions:

1. From earlier results it is expected that the $\mathrm{LNB}_{z t}$ model fits the observed data better, because it handles defect heterogeneity appropriately. For verification, the data sets are fitted to the $\mathrm{LNB}_{z t}$ and $\mathrm{Binom}_{z t}$ distributions in order to estimate the parameters for each data set. The goodness-offit is compared via AIC. Additionally, the binomial model is fitted with a Good-Turing adjustment.

2. From above considerations it is expected that the $\mathrm{LNB}_{z t}$ model yields the largest and most realistic estimates for remaining defects. Therefore, the total number of defects is estimated with each of the three models $-\mathrm{Bin}_{z t}, \mathrm{LNB}_{z t}$ and binomial with Good-Turing adjustment $\mathrm{Bin}_{G T}$. It is also expected that the estimation of remaining defects is reasonably precise (with a small confidence interval).

3. Finally, it is expected that the estimation of total number of defects is mostly unbiased for smaller process sizes and that reliability (narrowness of confidence intervals) increases with process size. Therefore, the total number of defects is estimated for several smaller process sizes (for $\mathrm{LNB}_{z t}$ only) and compared to the estimates on the complete data sets.

Please note, that the data analysis usually refers to the total number of defects $\hat{d}$, instead of the remaining number of defects $\hat{d}^{=0}$. This turned out being easier to depict in the tables and graphs. The remaining number of defects can always be obtained by substracting the known number of defects already discovered in an individual sample.

\section{Data Sets}

Four data sets go into the analysis. Three of these were previously used by Lewis to assess the adjustment terms for small sample sizes [8] and by Schmettow to prove existence of defect heterogeneity [13]: The two data sets MANTEL and SAVINGS stem from a publication assessing the performance of the Heuristic Evaluation [12]; the set MACERR is the result of a testing study [8] without thinking-aloud. The fourth data set EDU3D is from a comparison of two methods for identifying usability defects in an educational application with a 3D interface [1]. The methods under comparison were usability testing $(n=10)$ and a document based inspection using guidelines for ergonomic 3D interfaces $(n=10)$. Both conditions have been merged for the analysis here. Table 1

Table 1. Usability evaluation data sets under examination

\begin{tabular}{lcccc}
\hline Data Set & Type & Sessions & Defects $d^{+}$ & Ref \\
\hline EDU3D & UT, DI & 20 & 119 & {$[1]$} \\
MACERR & User Test & 15 & 145 & {$[8]$} \\
MANTEL & HE & 76 & 30 & {$[12]$} \\
SAVINGS & HE & 34 & 44 & {$[12]$} \\
\hline
\end{tabular}


Table 2. Fitting four data sets to three different models: parameter estimates ( $\left.\hat{p}, \hat{\mu}, \sigma^{2}\right)$, goodness-of-fit criterion (AIC), estimated total number of defects $(\hat{d})$ with confidence intervals $\left(d_{5 \%}, d_{95 \%}\right)$

\begin{tabular}{|c|c|c|c|c|c|c|c|c|c|c|c|c|c|c|c|}
\hline \multirow[t]{2}{*}{ Set } & \multicolumn{5}{|c|}{$\operatorname{Bin}_{z t}$} & \multicolumn{6}{|c|}{$\mathrm{LNB}_{z t}$} & \multicolumn{4}{|c|}{$\operatorname{Bin}_{G T}$} \\
\hline & $\hat{p}$ & AIC & $d_{5 \%}$ & $\hat{d}$ & $d_{95 \%}$ & $\hat{\mu}$ & $\hat{\sigma}^{2}$ & AIC & $d_{5 \%}$ & $\hat{d}$ & $d_{95 \%}$ & $\hat{p}$ & $d_{5 \%}$ & $\hat{d}$ & $d_{95 \%}$ \\
\hline EDU3D & .23 & 803 & 119 & 120 & 121 & -2.12 & 1.46 & 571 & 136 & 155 & 196 & .18 & 120 & 121 & 124 \\
\hline MACERR & .15 & 609 & 153 & 159 & 170 & -4.24 & 3.62 & 471 & 273 & 449 & 995 & .10 & 167 & 178 & 192 \\
\hline MANTEL & .38 & 1113 & 30 & 30 & 30 & -0.85 & 1.90 & 257 & 30 & 31 & 33 & .37 & 30 & 30 & 30 \\
\hline SAVINGS & .28 & 466 & 44 & 44 & 44 & -1.27 & 1.16 & 280 & 44 & 46 & 49 & .27 & 44 & 44 & 44 \\
\hline
\end{tabular}

gives an overview on the data sets; please note that $d^{+}$is the total number of defects that have been identified in each study. Due to omissions the true number of existing defects may be larger, but is unkown at first.

\section{Comparing the Binomial and LNB distributions}

First, all four data sets are fitted to the three models $\mathrm{Bin}_{z t}$ and $\mathrm{LNB}_{z t}$ and $\mathrm{Bin}_{G T}$. A maximum likelihood estimation is performed for each data set under each model. The value of the maximized likelihood function is used to calculate the AIC, which allows comparison of model fit, where a smaller AIC means a better fitting model. The Bin $_{G T}$ model adds virtual undiscovered defects for smoothing, which renders the AIC value not comparable. The results are reported in table 2: For all four data sets the $\mathrm{LNB}_{z t}$ model has a smaller $\mathrm{AIC}$ and is thus the preferred model.

The first two data columns in the table show the parameter estimates for each model. The values for $p$ and $\mu$ vary a lot between studies, which resembles the results of Nielsen and Landauer [11]. For the $\mathrm{LNB}_{z t}$ model considerable values for the variance parameter $\sigma^{2}$ are observed. This conforms with previous findings [13] showing that defect visibility not only varies between but also inside studies. Whereas the variance of defect visibility ranges between 1.16 to 1.90 for three data sets it is exceptionally large for MACERR together with a very low mean. This data set appears a little outlying compared to the others, whereas this is not so strongly reflected with the binomial estimators. For the smaller data sets EDU3D and MACERR the GT adjustment results in considerable lower estimates for $p$. This is different for the two larger data sets indicating that these are saturated - nearly all defects have been discovered.

\section{Determining the true number of defects}

Now, the likely total number of defects in each study is determined. Again, the results for all three models are reported in order to stress the difference. However, the $\mathrm{LNB}_{z t}$ model showed a better fit on the data and thus can be taken to yield estimators of better accuracy for the number of remaining defects $\hat{d}^{=0}$. Formula 7 is used to calculate the most likely value for $\hat{d}^{=0}$ and an additional bootstrapping analysis with 1000 samples is conducted to determine the 5\% and $95 \%$ confidence limits (constituting a $90 \%$ confidence interval).

Table 2 shows the results. As expected, the $\mathrm{LNB}_{z t}$ model estimates a larger number of overall existing defects $\hat{d}$ than
$\mathrm{Bin}_{z t}$ in all cases. Even for the large data sets MANTEL and SAVINGS it suggests that still a few defects remain undiscovered. The absolute difference to the binomial prediction is rather small which is due to these two processes being nearly saturated. The difference between the three models is particularly evident with the two smaller data sets. While the $\mathrm{Bin}_{z t}$ estimator tells that there is only one defect undiscovered in EDU3D the $\mathrm{LNB}_{z t}$ estimates that at least 11 defects are missing $\left(d_{5 \%}\right)$, but most likely 13 , and 36 if one wants to be sure $\left(d_{95 \%}\right)$. The results for the MACERR data set are again extreme. The $\mathrm{Bin}_{z t}$ model estimates up to 23 remaining defects $\left(d_{95 \%}\right)$, whereas the $\mathrm{LNB}_{z t}$ tells that only one-third of all defects have been discovered so far. This data set also shows the by far largest uncertainty with wide confidence intervals.

The $\operatorname{Bin}_{G T}$ estimators are usually somewhere in between, but closer to $\mathrm{Bin}_{z t}$. It seems that the GT adjustment provides more than enough smoothing for unseen events and thus to some extent compensates for varying defect visibility.

Due to the mathematical considerations and the better model fit the LNB model is to prefer. This truly makes a difference: The Binomial model consistently estimates a smaller number of remaining defects, irrespectively whether zero-truncation or GT adjustment is applied. Consider, for example, the EDU3D data set: 31 defects, which is more than a quarter, have been discovered only once and the binomial probability is small, too. It appears very unlikely, that there remain only two or three defect undiscovered. In contrast, the result from the $\mathrm{LNB}_{z t}$ model -26 are missing - is more realistic.

\section{Total number of defects at various process sizes}

The following analysis investigates how well the $\mathrm{LNB}_{z t}$ model predicts the number of remaining defects with smaller process sizes. Again, the bootstrapping procedure is employed in the following way: For each process size $p s$ the according number of sessions is randomly chosen from the data set. The margin sum of at least once discovered defects is calculated and drawing with replacement is applied to the margin sum. The latter may seem redundant, but is required for larger process sizes, where the first sampling step may not yield enough variation.

For each margin sum a maximum likelihood estimation was performed, resulting in up to 500 estimations for the total number of defects $\hat{d}_{p s}$. Occasionally, the numerical fitting procedure returns with an error, esp. for small process sizes. 

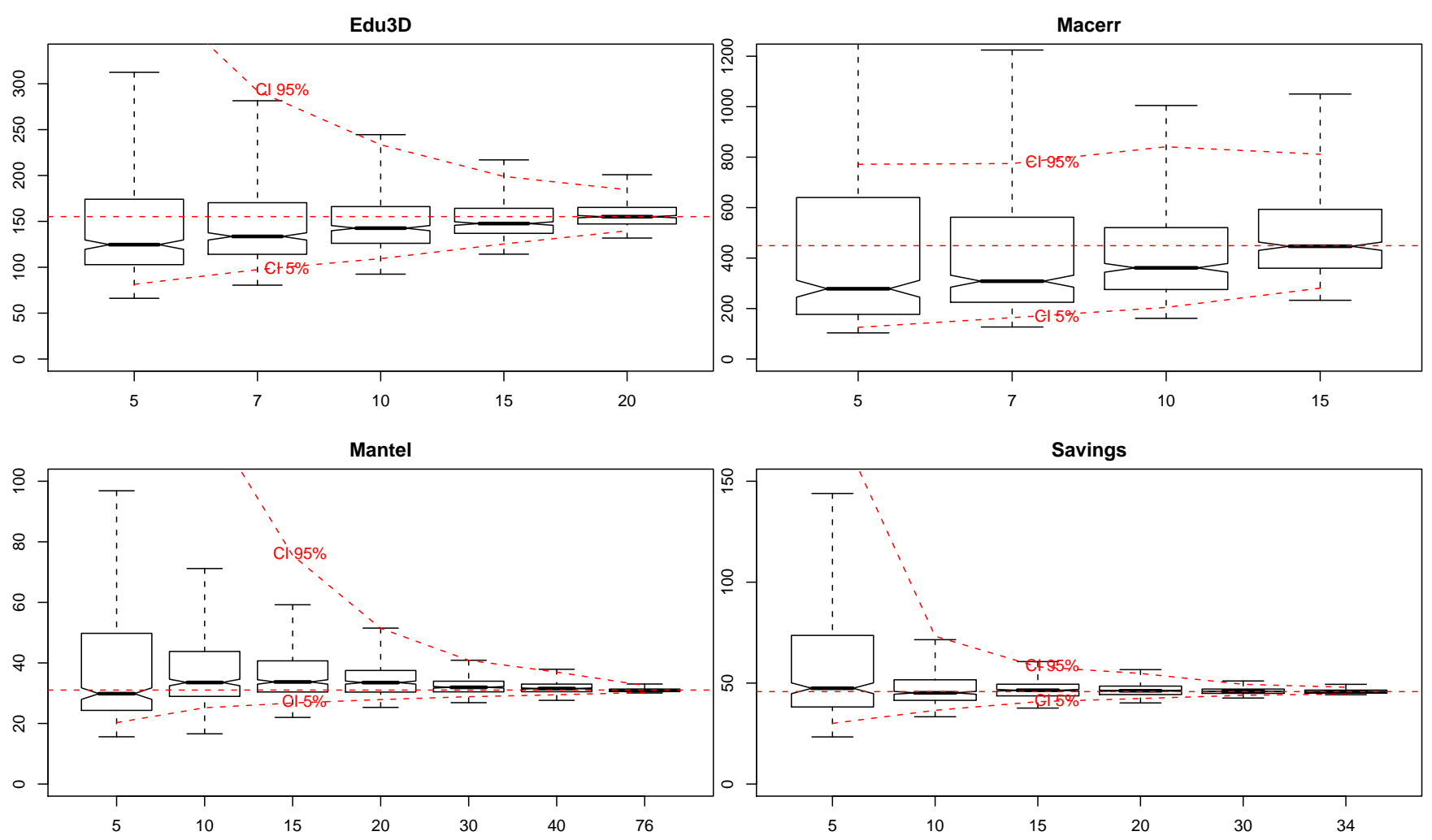

Figure 3. Estimated total number of defects with increasing sample sizes (horizontal dotted line is $\hat{d}$ with maximum sample size) MANTEL and SAVINGS match accurately, EDU3D has a small bias at small process sizes, estimates of MACERR are unusable.

This lengthy data analysis was run unsupervised, so these cases could only be removed afterwards. This may seem to restrict the practical utility or even validity of this kind of

Table 3. Estimating the total number of defects with small sample sizes: Average estimation and upper and lower limits

\begin{tabular}{crrrr}
\hline Data Set & size & $d_{5 \%}$ & $\bar{d}$ & $d_{95 \%}$ \\
\hline EDU3D & 5 & 84 & 166 & 615 \\
& 7 & 99 & 157 & 340 \\
& 10 & 112 & 154 & 243 \\
& 15 & 126 & 155 & 217 \\
\hline MACERR & 5 & 131 & 295 & 917 \\
& 7 & 173 & 353 & 932 \\
& 10 & 219 & 402 & 934 \\
\hline MANTEL & 5 & 20 & 58 & 193 \\
& 10 & 24 & 53 & 104 \\
& 15 & 26 & 41 & 65 \\
& 20 & 27 & 37 & 50 \\
& 30 & 29 & 33 & 40 \\
& 40 & 29 & 32 & 37 \\
\hline SAVINGS & 5 & 30 & 71 & 183 \\
& 10 & 37 & 51 & 77 \\
& 15 & 41 & 48 & 58 \\
& 20 & 42 & 47 & 54 \\
& 30 & 44 & 46 & 49 \\
\hline
\end{tabular}

data analysis. In fact, it is not problematic: Repeating the estimation procedure with different (e.g. randomly chosen) starting values will in most cases yield results. But, due to the random sampling there may still happen rare cases with extreme values, especially the margin sum having a very large proportion of once detected defects. One would never expect reasonable results from such data and instead go on with the study until the results become sufficiently expressive. An interesting observation is that by far the most failures happened with the MACERR (109 of 500 at $p s=5$ ). This is another hint for this data set being problematic in some way.

Table 3 shows the results which are also illustrated as boxplots with $25 \%$ quantiles (and outliers removed) in figure 3. First, we observe that for the data sets MANTEL and SAVINGS the median is rather consistent even for small process sizes. In contrast, EDU3D shows some underestimation of total number of defects for smaller process sizes. This slight bias is not very critical: The mean number of identified defects for EDU3D are 74 for $p s=5$ and 97 for $p s=10$, which is considerably lower than the median of estimated total defects at these sizes - thus, there is not really a risk of finishing the study to early.

Second, we see that in general the confidence intervals tighten with increasing process size, although this varies with the data sets. With 15 sessions, EDU3D and SAVINGS are already in a useful range, where $d_{95 \%}$ exceeds $\hat{d}$ by less than one-third. 
MANTEL reaches this criterion with 30 sessions. Comparing this to the parameter estimates in table 2 it seems that two factors influence the reliability of $\hat{d}$ : When $\mu$ is larger (SAVINGS) or variance $\sigma^{2}$ is smaller (SAVINGS and EDU3D) sufficient reliability is obtained with smaller process sizes.

In contrast, MACERR is extreme in both aspects and again appears problematic: The upper confidence limit even increases with more sessions and is still useless at 15 sessions. Furthermore, there is a stronger bias to underestimation, which also shows up in the means reported in table 3 . The large confidence intervals may be partly due to the very low detection probability, which causes samples with low information (e.g. most of the defects been discovered only once or twice). But, there may be further explanations for the irregular behavior; this topic is returned to in the discussion section.

Note, that table 3 also shows the average estimated number of defects $\bar{d}$. This appears to be larger for small sample sizes (except for MACERR). This is neither a true bias in the model nor a paradox. The cause lies in the asymmetric (skewed) distribution of $\hat{d}$. Only for symmetric (and of course unimodal) distributions the most likely point is exactly the mean. In fact, this skewed distribution of $\hat{d}$ is the reason why bootstrapping the confidence intervals was chosen here instead of relying on the asymptotic normality of maximum likelihood estimators. In any case, these are complications relevant for evaluating such models which is done here. They will not bother the practitioner who is applying an evaluated model with well prepared statistical tools.

\section{A RUNNING EXAMPLE}

The mathematical background of the approach introduced here is not trivial. However, the actual procedure of estimation is supported by statistical programs the author has written. These are freely available on an accompanying website [14]. The following example illustrates how to practice quantitative control of usability studies. This example uses the data from the SAVINGS data set, pretending it were from a usability testing study. From the previous analysis it is known that there were most likely 46 defects, 44 of these have been discovered with 34 session.

Imagine a usability consulting company that was just hired for a formative usability testing study on a newly designed e-commerce platform. Because the customer is aware that usability is mission-critical, the contract includes a claim that the study must at least discover $90 \%$ of existing usability defects (approximately 42). From previous experience the study manager guesses that around 20 testing session are required. However, she decides to not rely on experience, instead do controlling with the $\mathrm{LNB}_{z t}$ approach and sets a strict confidence limit of $95 \%$. After 8 sessions, having identified 36 defects, she runs an initial data analysis. The $\mathrm{LNB}_{z t}$ estimates are $\mu=-.76$ and $\sigma^{2}=1.09$ and $\operatorname{LNB}(0,8,-.76,1.09)=$ .13. This gives an estimation of $\hat{d}=36 / 1-.13=41$ which appears pretty close to the number of defects discovered so far. However, bootstrapping reveals an upper $95 \%$ confidence limit of $d_{95 \%}=61$. This would require to discover 55 defects in order to meet the confidence criterion. The manager decides to analyze whether the required number of sessions is in the planned range and uses the two estimators with the LNB version of formula 9. She finds that with the current parameters 10 sessions yielded a coverage of $90 \%$ $(1-\operatorname{LNB}(0,10,-.76,1.09)=.90)$. and concludes that with approximately 10 sessions the goal may be reached. However, the confidence limit has to be regarded as well. The bootstrap also yielded the distribution of parameters. Taking the $95 \%$ "pessimistic" values for $\mu$ and $\sigma^{2}$ the $90 \%$ goal may also happen after 28 sessions.

The project manager proceeds with doing this analysis after each four new sessions until 20 session and the criterion starts approaching the goal. Finally, after another two sessions 42 defects have been discovered and the upper confidence limit reaches 46 which now suffices, because $42 / 46=91 \%$. The complete results are shown in table 4 . Retrospectively, the project manager notices that she had reached the goal with 16 sessions already. The extra six sessions is the price to pay for being $95 \%$ sure that contract obligations are met.

Table 4. Example of controlling an evaluation study

\begin{tabular}{ccccc}
\hline Process Size $p s$ & Discovered $d_{p s}$ & $\hat{d}$ & $d_{95 \%}$ & $d_{p s} / d_{95 \%}$ \\
\hline 8 & 36 & 41 & 61 & $59 \%$ \\
12 & 41 & 45 & 54 & $76 \%$ \\
16 & 42 & 45 & 51 & $82 \%$ \\
20 & 42 & 44 & 48 & $88 \%$ \\
22 & 42 & 44 & 46 & $\mathbf{9 1 \%}$ \\
\hline
\end{tabular}

\section{DISCUSSION}

This work aims at establishing reliable quantitative control for formative usability evaluation processes. It bases on previous findings that accounting for varying defect visibility is required to prevent harmfully overoptimistic estimations. The $\mathrm{LNB}_{z t}$ model is a little beyond curricular statistics, but still it has a natural interpretation - defect visibility being a normally distributed latent property that manifests in the number of successful identifications. Arguably, assuming the normal distribution for this visibility property lacks empirical evidence, but this is not crucial as the mathematical model can be adapted to other distribution types (in case someone shows that the normality assumption is severely violated). Furthermore, this model applies to the powerful maximum likelihood method with related concepts such as model selection and confidence intervals. This is a particular advantage compared to the GT smoothing of the binomial model.

Confidence intervals have been widely ignored in previous studies on measuring and predicting evaluation processes; usually these reported on point estimators only. Here, the bootstrapping method is introduced to obtain proper confidence intervals. This accounts for the inherent randomness of statistical estimators, such as the model parameters and the number of remaining defects. Relying on point estimators may be good enough for internal tracking of studies, but confidence intervals allow for giving provable guarantees to customers of evaluation studies.

The proposed model is shown to fit the data sets significantly 
better than the binomial model. Thus, there is reason to believe the results being much closer to the truth. This really makes a difference when the total (or remaining) number of defects is at question. The analysis on the complete data sets shows that in each study a certain number of defects remained undiscovered.

In the evaluation study for three out of four data sets the estimation is sufficiently accurate at moderate process sizes. In contrast, the confidence intervals are very large for small samples. This raises severe doubts whether projection from early stages of a study are reliable, as was suggested by Lewis. In turn, as the confidence intervals sufficiently tighten with 15 sessions, "late" control of evaluation studies towards a preset goal seems feasible. Still, the running example based on the SAVINGS data set shows that reliable quantitative control comes at some costs: Although few new defects are discovered late in a study, it may often be required to continue testing in order to reach the preset confidence criterion. However, for the author this appears in an acceptable range when high reliability is at stake.

More severe concerns are raised by looking at the results on the MACERR data set: The estimation of total defects was rather extreme and the confidence intervals literally refused to diminish. An amazing fact with this data set is the very large proportion of defects detected only once being more than a half $\left(d^{=1}=76\right)$. Still, there are also a few defects that have been discovered very often ( 15 have been discovered more than five times), which illustrates the strong variation in defect visibility. As shown in figure 4 the fitted distribution tightly renders the observed data points. But, as a result of high variance and large number of once detected defects, it has a strong positive skewness predicting 304 remaining defects. The author is not aware of any usability study that dealt with such a high number of defects, neither in the literature nor in own practice. But, there are two possible explanations for the irregularity of the data set:

First, the data set may contain a larger number of highly subjective defect reports. This phenomenon was first described by Sears [17] and has been referred to as false alarms in the current HCI literature (e.g. [4]. Typically, those reports are not shared by other observers or experts and thus appear rarely. However, the MACERR data set has been obtained by usability testing which is usually held to be robust against false alarms. (In fact, it is the recommended approach to identify them [19]).

Second, the individual defect reports may not have been thoroughly aggregated by proper matching, leaving multiple identical defects as distinct data points in the data set with a low frequency of occurrence. Recently, this topic has been closely examined by Hornbæk and Frøkjær, showing that the results of different matching techniques differ substantially [5]. In particular, they found that certain matching techniques produce poor results, leaving many similar defect reports as distinct defect types in the data sets.

These considerations are hypothetical regarding the strange

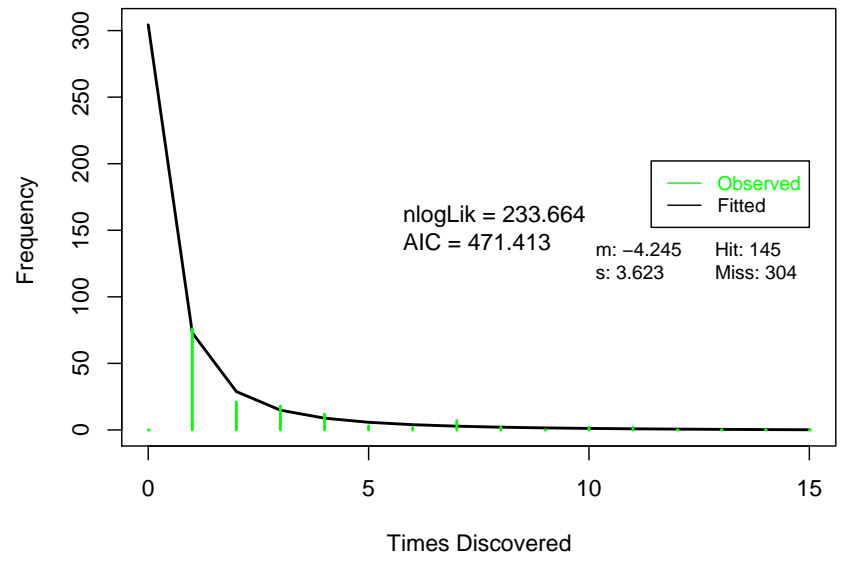

Figure 4. Strong variance and a large number of once detected defects in MACERR cause a extreme skew of the LNB distribution

behavior of the MACERR data set; nevertheless they are crucial for the practice of quantitative study control. In order to get reliable results with the approach shown here, it is essential to prevent high false alarm rates and to apply a proper and thorough data aggregation. Most likely, this causes extra costs for conducting such high reliability studies in industrial practice.

In any case, the two other models $\mathrm{Bin}_{z t}$ and $\mathrm{Bin}_{G T}$ do not show such extreme results with the MACERR data set. This does not allow the conclusion of them having a better reliability in such cases. These two models simply ignore the apparent variation in defect visibility. The larger this variation is, the stronger is the optimistic bias.

There also is another interesting result: The data set EDU3D was a merge of two conditions with different evaluation methods. The qualitative analysis of Bach and Scapin shows that these two methods differ widely in the types of defects they support better [1]. There already are some reports on such different method profiles in the literature (e.g. [3] [15]), but this topic still needs further examinations. Notably, this is a kind of heterogeneity different to defect visibility, it is a heterogeneity in sessions. And possibly, the small bias at early process sizes of EDU3D is due to session heterogeneity. This points to an interesting research questions for future studies: How do different groups of participants and mixed-method processes perform in usability studies? Further examinations on the EDU3D data set (and similar ones) may clarify such questions, but this probably requires further advances in statistical models for the usability evaluation process. In any case, this would also be an important step towards better understanding the capabilities of usability evaluation methods.

\section{CONCLUSION}

Varying defect visibility decelerates the evaluation process which causes the former geometric series model to underestimate the remaining number of defects. The zero-truncated logit-normal-binomial model was introduced and shown to perform well for estimating the number of remaining defects. 
This allows practitioners to control evaluation studies towards a preset goal. The same approach also applies for estimating the number of required sessions from early data. This was the main tenor of previous research, but has to be taken with great care - there is severe uncertainty with small sample sizes. Taking confidence intervals into account is strongly advised.

Quantitative control of usability studies comes at some costs: A larger number of sessions is required to narrow the confidence interval of estimated remaining defects. Additional costs are due to careful data preparation, especially defect matching. This may not be justifiable for the majority of "discount" evaluation projects. But, there are cases where usability is mission- or even safety-critical. The author's rough estimate is that high-reliability studies have about double the costs. This appears much on the first glance, but may be justified by economical or other risks. Usability agencies may start thinking about high-reliability evaluations studies as part of their service portfolio. The author sees this as an interesting field of research and is willing to assist.

\section{ACKNOWLEDGMENT}

The author wishes to thank all previous authors who have published their complete data sets. Special thanks go to Cédric Bach for sharing the high quality data set EDU3D.

\section{REFERENCES}

1. C. Bach and D. L. Scapin. Comparing inspections and user testing for the evaluation of virtual environments. International Journal of Human-Computer Interaction. in review.

2. Kenneth P. Burnham and David R. Anderson. Multimodel inference. understanding AIC and BIC in model selection. Sociological Methods \& Research, 33(2):261-304, 2004.

3. Erik Frøkjær and Kasper Hornbæk. Metaphors of human thinking for usability inspection and design. ACM Trans. Comput.-Hum. Interact., 14(4):1-33, 2008.

4. H. Rex Hartson, Terence S. Andre, and Robert C. Williges. Criteria for evaluating usability evaluation methods. International Journal of Human-Computer Interaction, 15(1):145-181, 2003.

5. Kasper Hornbæk and Erik Frøkjær. Comparison of techniques for matching of usability problem descriptions. Interacting with Computers, 20:505-514, 2008 .

6. F. Lad and P. Frederic. Two moments of the logitnormal distribution. Communications in Statistics: Simulation and Computation, 37(7):in print, 2008

7. J. R. Lewis. Sample sizes for usability studies: Additional considerations. Human Factors, 36:368-378, 1994.

8. James R. Lewis. Evaluation of procedures for adjusting problem-discovery rates estimated from small samples. International Journal of Human-Computer Interaction, 13(4):445-479, 2001.
9. George P. Moore, David S. McCabe. Introduction to the Practice of Statistics, chapter Bootstrap Methods and Permutation Tests. W.H. Freeman \& Co, 5th edition, 2005.

10. Jakob Nielsen. Usability Engineering. Morgan Kaufmann, San Diego, 1993.

11. Jakob Nielsen and Thomas K. Landauer. A mathematical model of the finding of usability problems. In CHI '93: Proceedings of the SIGCHI conference on Human factors in computing systems, pages 206-213, New York, NY, USA, 1993. ACM Press.

12. Jakob Nielsen and Rolf Molich. Heuristic evaluation of user interfaces. In CHI '90: Proceedings of the SIGCHI conference on Human factors in computing systems, pages 249-256, New York, NY, USA, 1990. ACM.

13. Martin Schmettow. Heterogeneity in the usability evaluation process. In David England and Russell Beale, editors, Proceedings of the HCI 2008, volume 1 of People and Computers, pages 89-98. British Computing Society, 2008.

14. Martin Schmettow. Controlling the usability evaluation process - accompanying website. Website, March 2009. http://schmettow.info/Controlling.

15. Martin Schmettow and Sabine Niebuhr. A pattern-based usability inspection method: First empirical performance measures and future issues. In Devina Ramduny-Ellis and Dorothy Rachovides, editors, Proceedings of the HCI 2007, volume 2 of People and Computers, pages 99-102. BCS, September 2007.

16. Martin Schmettow and Wolfgang Vietze. Introducing Item Response Theory for measuring usability inspection processes. In $\mathrm{CHI}$ '08: Proceeding of the twenty-sixth annual SIGCHI conference on Human factors in computing systems, pages 893-902, New York, NY, USA, 2008. ACM.

17. Andrew Sears. Heuristic walkthroughs: Finding the problems without the noise. International Journal of Human-Computer Interaction, 9(3):213-234, 1997.

18. Robert A. Virzi. Refining the test phase of usability evaluation: How many subjects is enough? Human Factors, 34(4):457-468, 1992.

19. Alan Woolrych, Gilbert Cockton, and Mark Hindmarch. Falsification testing for usability inspection method assessment. In Proceedings of the HCIO4 Conference on People and Computers XVIII, 2004. 